Journal of Animal and Veterinary Advances 11 (7): 1046-1051, 2012

ISSN: $1680-5593$

(C) Medwell Journals, 2012

\title{
Prokaryotic Expression and Sequence Analysis of Porcine BCL10 Gene
}

\author{
Zhicai Zuo, Shan Liao, Xinqiao Qi, Yue Yi, Zhiwen Xu, Ling Zhu, \\ Wanzhu Guo and Hengmin Cui \\ College of Veterinary Medicine, Sichuan Agricultural University, \\ Ya'an, 625014 Sichuan, China
}

\begin{abstract}
BCL-10 is an important functional and an apoptotic regulator gene inducing the organismic innate immunity. It has not been reported that the BCL-10 protein of porcine in normal level could motivate immune factor to produce. Bioinformatics analysis was performed to predict the characteristics of BCL-10, the results indicated that $B C L-10$ gene encoded a polypeptide, molecular mass of $45 \mathrm{Ku}$ and comprising 233 amino acids. The prediction of secondary structure showed that BCL-10 had 9 alpha-helix, 1 beta-sheet, 12 turn and 14 random coil. The protein had twenty transmembrane regions, an CARD structure field which located in 15-100 amino acids and no signal peptide, the hydrophobic index was between -2.500 and 1.400 . The phylogenetic tree showed that BCL-10 was similar to human (the homology index was $>95 \%$ ) and had a distant relationship to mouse. To identify the activity of BCL-10, the protein with a His-tag was expressed in $E$. coli Rosetta and purified. The purified protein showed an obvious effect of cell proliferation. Cloning and analysising of $B C L-10$ gene which laid foundation to further research and exploitation.
\end{abstract}

Key words: BCL-10, molecular cloning, Bioinformatics, Prokaryotic expression, human, China

\section{INTRODUCTION}

$B C L-10$ is an important functional and apoptotic regulator gene inducing the organismic innate immunity which is firstly cloned from the chromosomes $\mathrm{t}(1 ; 14)$ (p22; q32) of the demic MALT lymphomas (Zhang et al., 1999; Sagaert et al., 2007). BCL-10 gene encoded a polypeptide, comprising 233 amine acids and the Nterminal has a CARD structural domain which is similar to RICK and ARC activating the NF- $\mathrm{kB}$ to participate the organismic innate immunity and inducing apoptosis of the cell (Briones et al., 2009; Wegener and Krappmann, 2007; Bhattacharyya et al., 2009).

The BCL-10 protein in normal can motivating immune factor to produce, enhancing the innate immunity and preventing the infection of the pathogen like bacteria and virus but if the protein is over expressed which will inhibit apoptosis and promote cell to transform not to apoptosis, especially promote the lymphocytes to malignantly transform to the MALT lymphoma (Courts et al., 2007; Jost and Ruland, 2007). Many studies on the BCL-10 concentrate upon demic MALT lymphomas but the researches about the porcine BCL-10 are very limited. In this study, $B C L-10$ gene was cloned from porcine PMBC by RT-PCR, the prokaryotic expression vector was constructed and the structure of the BCL- 10 protein was analyzed by bioinformatics methods. As well as the BCL10 protein can be highly expressed in Escherichia coli.
This research laid a foundation for the study of the function of the porcine BCL-10 and the biologic activity of the BCL-10 protein.

\section{MATERIALS AND METHODS}

The Plasmid PET32a (+), E. coli Rosetta and E. coli $\mathrm{DH} 5 \alpha$ were provided by Key Laboratory of Animal Diseases and Human Health of Sichuan province. RNAiso reagent, Prime Script RT reagent kit, PMD19-T simple trage, $2 \times$ Taq PCR Master Mix, restriction endonuclease EcoRI, XhoI and T4 DNA ligase were purchased from TaKaRa. Plasmid Mini Kit were purchased from OMEGA. Murine monoclonal antibody of histidine were purchased from CST corporation; sheep anti-mouse IgG tagged with HRP and Bovine Serum Albumin (BSA) were purchased from JINGMEI Biotechnology Co. Ltd. ConA were purchased from WATSON Biotechnologies, Inc (Shanghai, China). RPMI1640 were purchased from HyClone corporation. Lydroxypropylmethyl cellulose were purchased from blood institutes (Tianjin, China). Other reagents were all from China-made determination of High-Parity. Referring Porcine $B C L-10$ gene embodied with GenBank accession no FJ376731.1, a pair of specific primers were designed by using Oligo 6.7 Software program. The forward Primer (P1) is 5'-GAATTCAT GGAGCCCGCCGCGCCGT-3' containing a EcoRI site (underlined) and the reverse primer (P2) is $5^{\prime}$-CTCGA

Corresponding Author: Wanzhu Guo, College of Veterinary Medicine, Sichuan Agricultural University, Ya'an, 625014 Sichuan, China 
GTCATTGCCGCAAAAGAGCACGT-3' with a XhoI site (underlined). Primers were synthesized at Invitrogen Corporation (Shanghai, China).

RNA extraction: Separating PMBC (Kovar et al., 2009) cultured from the healthy and weaned piglets' vena cava anterior blood in the sterile condition and cropping the lymphocytes 2 days latter induced by ConA was to extract the total lymphocytes cell RNA with the RNAiso Reagent according to the operating instruction. The samples were stored at $-70^{\circ} \mathrm{C}$ until use.

RT-PCR amplification: The complementary DNA ofIL-18 was synthesized with one-step RT-PCR kit (TaKaRa, Dalian, China). Reactions were performed at $95^{\circ} \mathrm{C}$ for $5 \mathrm{~min}$ followed by 35 cycles of $94^{\circ} \mathrm{C}$ for $30 \mathrm{sec}, 55^{\circ} \mathrm{C}$ for $45 \mathrm{sec}$ and $72^{\circ} \mathrm{C}$ for $45 \mathrm{sec}$ followed by $72^{\circ} \mathrm{C}$ for $10 \mathrm{~min}$. The amplified products was verified by $10 \mathrm{~g} \mathrm{~L}^{-1}$ agarose gel electrophoresis.

Cloning and sequencing: Resultant products were electrophoresed on $1 \%$ agarose gel containing ethidium bromide $\left(10 \mathrm{mg} \mathrm{mL}^{-1}\right)$. The amplified band corresponding to expected BCL-10 cDNA fragment was excised from the gel and purified with an Agarose Gel DNA Extraction kit (Tiangen). The purified cDNA fragments were ligated into pMD19-T cloning vectors (TaKaRa) and was named as pMD-BCL-10 then transformed into $E$. coli $\mathrm{DH} 5 \alpha$ cell and the recombinant plasmids were isolated and were sequenced in Invitrogen Corporation and the obtained sequences of porcine $B C L-10$ gene and the $B C L-10$ gene of human being, swine, mouse and other reference mammals were comparatively analyzed by using DNAMAN and DNA Star 7.0. After that the protein sequences of porcine $B C L-10$ gene was predicted and analyzed by NCBI BLASTP program and other on-line tool. Secondary structure was predicted by DNA Star 7.0; transmembrane region by TMpred (http://www.ch. embnet.org/software/TMPRED_form.html); Signal peptide cleavage sites by SignalP (http://www.cbs.dtu.dk/ services/SignalP/).

Prokaryotic Expression: Porcine BCL-10 was inserted into the vector PET32a $(+)$ by double digestion using EcoRI, XhoI to construct the recombinant expression plasmid PET32-BCL10 and then identified by enzyme digestion and PCR and the recombinant plasmids were isolated and sequenced in Invitrogen Corporation. After that the recombinant plasmids were transformed into competent E. coli Rosetta (DE3) accordingly for expression and induced $0 \sim 8 \mathrm{~h}$ (taking samples every $1 \mathrm{~h}$ ) with the induced concentration of IPTG $0,0.4,0.8,1.2,1.6$ and $2.0 \mathrm{mmol} \mathrm{L}^{-1}$, respectively and then choice the optimal induced concentration of IPTG and the optimal time.

Western-blot analysis of prokaryotic expression products: According to commonly used experimental methods of Immunology (Zhu and Chen, 2000). Firstly, transferred the bacterial liquid above into the cellulose membrane (NC) after analyzed by SDS-PAGE. Secondly sealed the cellulose membrane $(\mathrm{NC})$ with the $30 \mathrm{~g} \mathrm{~L}^{-1}$ Bovine Serum Albumin (BSA) then washed by TBST. Thirdly, added mouse histidine Monoclonal antibodies in 1:2000 and then incubated overnight. Followed that, added in sheep anti-mouse IgG tagged with HRP after washed by TBST and then were incubated $1 \mathrm{~h}$. Finally, Reacted by DAB method after washed.

Activity detection of prokaryotic expressed products: MTT assay was performed to evaluate the activity of prokaryotic expressed products. Prepared the cell suspension of porcine splenic lymphocytein under sterile condition for adding into the 96 hole plate, every group was consist of 4 holes each contained $2 \times 10 \times 5$ cells. Depurated and detected the activity of the protein according to the methods of the reference documents (Guo-Quan et al., 2009). Through the data of A570 compute, the cell proliferation index (proliferation index $=$ test team's A570/control group's A570).

\section{RESULTS}

RT-PCR amplification: The result showed that cloned BCL-10 cDNA was about $700 \mathrm{bp}$ agreed with theoretical calculation in Fig. 1 and 2.

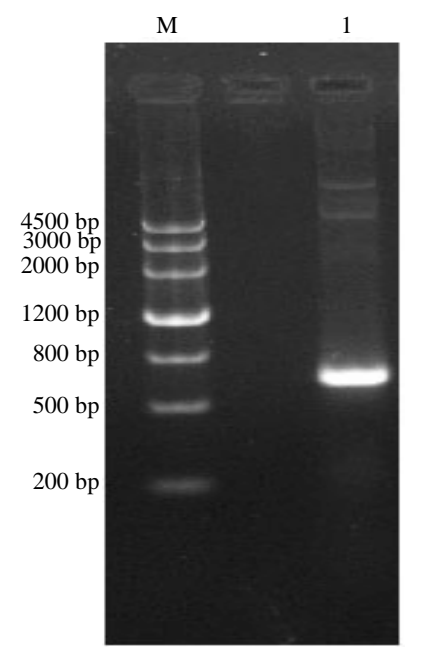

Fig. 1: Electrophoretic analysis of porcine, Lane M: Marker III. Lane 1: RT-PCR products 
The construction of phylogenetic tree based on thebcl-10 sequence of Giant Pandas and 1, 7 reference mammals: NCBI BLASTP program was used to perform nucleotide sequence homology searches.

The result showed that this $B C L-10$ gene had $99 \%$ homology of nucleotide with that of Porcine BCL-10 (accession no: FJ376731) and only 3 nucleotide mutation were different found in 350, 471 and 656 region. But compared with human and mouse, the homology at nucleotide acid level was 89 and $83 \%$, respectively. Phylogenetic analysis of Porcine $B C L-10$ gene compared with other 17 reference animals showed that it was similar to human and had a distant relationship to mouse (Fig. 3). On the other hand, the homology index of the amino acid sequence analysis was $>95 \%$ (Table 1).

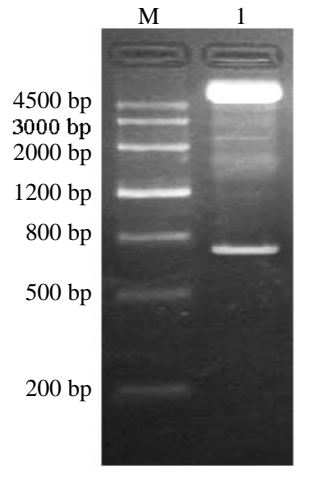

Fig. 2: Identification of the recombinant BCL10 gene amplified by RT-PCR plasmid PET32-BCL10, Lane M: MarkerIII. Lane 1: Products from digested PET32-BCL10

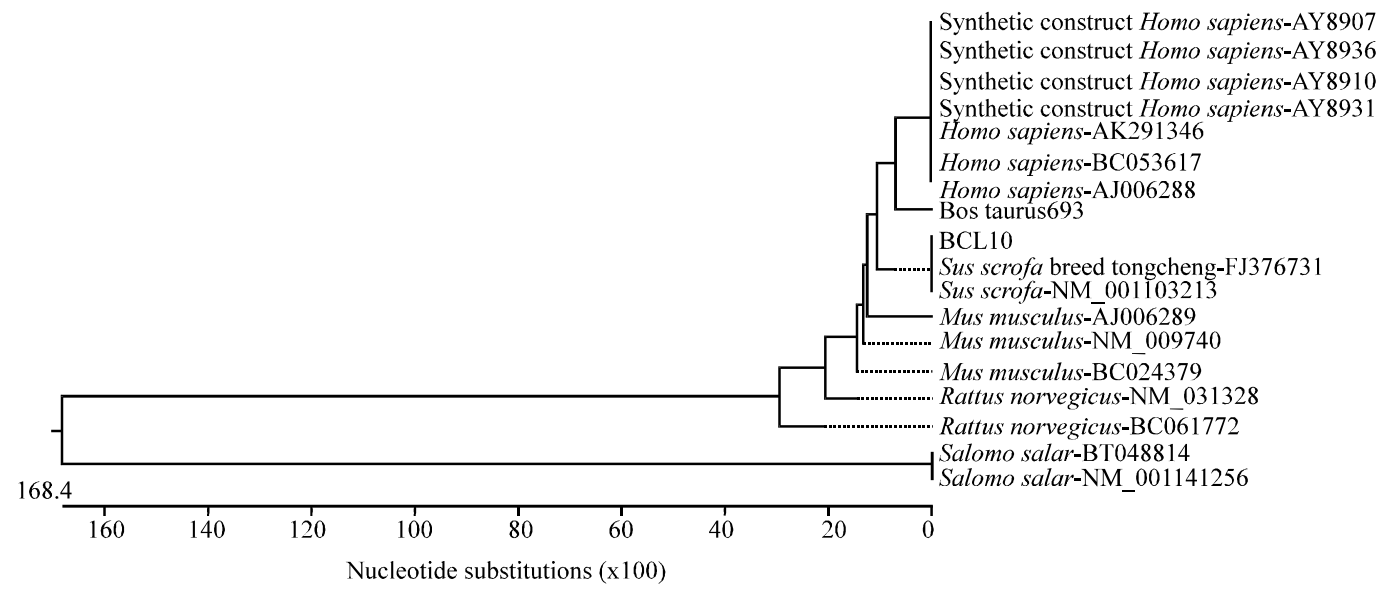

Fig. 3: Phylogenetic tree of $B C L-10$ gene encoding amino acid sequence

Table 1: Homology comparison of BCL-10 with that of other reported mammals at the aa level

\begin{tabular}{|c|c|c|c|c|c|c|c|c|c|c|c|c|c|c|c|c|c|c|}
\hline \multirow[b]{2}{*}{ Divergence } & \multicolumn{18}{|c|}{ Percent identity } \\
\hline & e 1 & 2 & 3 & 4 & 5 & 6 & 7 & 8 & 9 & 10 & 11 & 12 & 13 & 14 & 15 & 16 & 17 & 18 \\
\hline 1 & - & 85.9 & 89.7 & 89.6 & 89.6 & 82.9 & 82.9 & 82.9 & 82.8 & 82.8 & 26.4 & 26.4 & 100.0 & 100.0 & 89.2 & 89.2 & 89.3 & 89.2 \\
\hline 2 & 15.7 & . & 87.0 & 87.2 & 87.2 & 79.7 & 79.5 & 79.5 & 79.1 & & 25.1 & 25.1 & 85.9 & 85.9 & 87.0 & 87.2 & 7.2 & 87.0 \\
\hline 3 & 11.2 & 14.3 & . & 99.9 & 99.9 & 83.9 & 83.8 & 83.8 & 825 & 8 & 2 & 25.1 & 9.7 & 9.7 & 9.4 & 9.4 & 9.6 & 99.4 \\
\hline 4 & 11.3 & 14.1 & 0.1 & - & 100.0 & 84.0 & 83.9 & 83.9 & 82.6 & 82.6 & 25.3 & 25.3 & 89.6 & 89.6 & 6 & 99.6 & 9.7 & 99.6 \\
\hline 5 & 11.3 & 14.1 & 0.4 & 0.0 & - & 84.0 & 83.9 & 83.9 & 82.6 & 8 & 25.3 & 25.3 & 89.6 & 89.6 & 99.6 & 99.6 & 99.7 & 99.6 \\
\hline 6 & 19.6 & 23.9 & 18.3 & 18.1 & 18. & 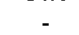 & 99.9 & 99.9 & 92.7 & 92. & 26.9 & 26.9 & 82.9 & 82.9 & 83.6 & 83.9 & 83.8 & 83.6 \\
\hline 7 & 19.6 & 24.1 & 18.5 & 18.3 & 18.3 & 0.1 & - & 100.0 & 92.9 & 92.9 & 26.9 & 26.9 & 82.9 & 82.9 & 5 & 83.8 & 3.6 & 83.5 \\
\hline 8 & 19.6 & 24. & 18.5 & 18.3 & 18.3 & 0. & 0.0 & - & 92.9 & 92.9 & 26.9 & 26.9 & 82.9 & & 83.5 & 83.8 & 3.6 & 83.5 \\
\hline 9 & 19.7 & 24.7 & 20.2 & 20.0 & 20.0 & 7. & 7.5 & 7. & - & 100.0 & 27.2 & 27.2 & 82.8 & 82.8 & 82.2 & 82.5 & 82.3 & 82.2 \\
\hline 10 & 19.7 & 24.7 & 20.2 & 20.0 & 20.0 & 7.7 & 7.5 & 7.5 & 0.0 & 0 & 27.2 & 27.2 & 82.8 & 82.8 & 82.2 & 82.5 & 82.3 & 82.2 \\
\hline 11 & 317.3 & 350.0 & 50.0 & 350.0 & 350.0 & 77.8 & 77.0 & 277.0 & 273.4 & 273 & - & 100.0 & 26.4 & 26.4 & 25.4 & 25.4 & 25.3 & 25.4 \\
\hline 12 & 317.3 & 350.0 & 350.0 & 350.0 & 350.0 & 277.8 & 277.0 & 277.0 & 273.4 & 273.4 & 0.0 & - & 26.4 & 26.4 & 25.4 & 25.4 & 25.3 & 25.4 \\
\hline 13 & 0.0 & & 11.2 & 11.3 & 11.3 & 19.6 & 19.6 & 19.6 & 19.7 & 19.7 & 317.3 & 317.3 & - & 100.0 & 89.2 & 89.2 & 89.3 & 89.2 \\
\hline 14 & 0 & & 11.2 & 11.3 & 11.3 & & & 19. & 10 & 19.7 & 317.3 & 317.3 & 0.0 & - & 89.2 & 89.2 & 89.3 & 89.2 \\
\hline 15 & 11.9 & 14.3 & 0.6 & 0.4 & 0.4 & 18.7 & 18.9 & 18.9 & 20.6 & 20.6 & 350.0 & 350.0 & 11.9 & 11.9 & - & 99.7 & 99.7 & 100.0 \\
\hline 16 & 11.9 & 14 & 0.6 & 0.4 & 0.4 & 18.3 & 18.5 & 18.5 & 20.2 & 20.2 & 350.0 & 350.0 & 11.9 & 11.9 & 0. & - & 99.7 & 99.7 \\
\hline 17 & 11. & & 0.4 & 0. & 0. & 18. & 18. & 18. & 20. & 20.4 & 350.0 & 350.0 & 11.7 & 11.7 & 0. & 0.3 & . & 99.7 \\
\hline 18 & 11.9 & 14.3 & 0.6 & 0.4 & 0.4 & 18.7 & 18.9 & 18.9 & 20.6 & 20.6 & 350.0 & 350.0 & 11.9 & 11.9 & 0.0 & 0.3 & 0.3 & 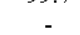 \\
\hline
\end{tabular}

1: BCL10; 2: Bos tourus693; 3: Homo sopiens-AJ006288; 4: Homo sapiens-AK291346; 5: Homo sapiens-BC053617; 6: Mus musculus-AJ006289; 7: Mus musculus-BC024379; 8: Mus musculus-NM_009740; 9: Rattus norvegicus-BC061772; 10: Rattus norvegicus-NM_031328; 11: Salomo salcor-BT048814; 12: Salomo salar-NM_001141256; 13: Sus scrofa breed tongcheng-FJ376731; 14: Sus scrofa-NM_001103213; 15: Synthetic construct Homo sapiens-AY8907; 16: Synthetic construct Homo sapiens-AY8910; 17: Synthetic construct Homo sapiens-AY8931; 18: Synthetic construct Homo sapiens-AY8936 


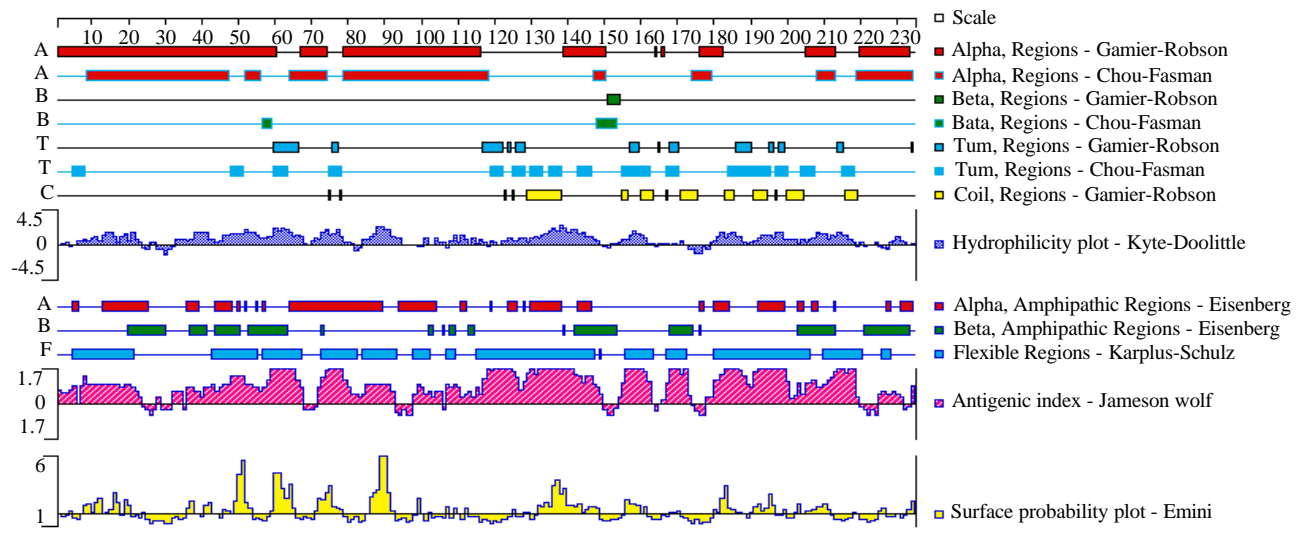

Fig. 4: Secondary structure prediction of porcine BCL-10

\begin{tabular}{lccc} 
Table 2: The composition of amino acid encoded by porcine $B C L-10$ gene \\
\hline & Whole protein composition analy sis \\
& - Number $^{-}$ & $\begin{array}{c}\text { Percentage by } \\
\text { weight }\end{array}$ & $\begin{array}{c}\text { Percentage by } \\
\text { frequency }\end{array}$ \\
Amino acid (s) & count & 42.66 & 36.05 \\
Charged (RKHYCDE) & 84 & 18.14 & 16.31 \\
Acidic (DE) & 38 & 18.07 & 14.06 \\
Basic (KR) & 33 & 23.86 & 26.18 \\
Polar (NCQSTY) & 61 & 27.40 & 28.33 \\
Hydrophobic (AJFWV) & 66 & 2.98 & 4.72 \\
A Ala & 11 & 1.97 & 2.15 \\
C Cys & 5 & 4.83 & 4.72 \\
D Asp & 11 & 13.31 & 11.59 \\
E Glu & 27 & 4.49 & 3.43 \\
F Phe & 8 & 2.18 & 4.29 \\
G Gly & 10 & 2.62 & 2.15 \\
H His & 5 & 3.89 & 3.86 \\
L lle & 9 & 7.34 & 6.44 \\
K Lys & 15 & 13.39 & 13.30 \\
L Lue & 31 & 1.00 & 0.86 \\
M Met & 2 & 3.92 & 3.86 \\
N Asn & 9 & 6.67 & 7.73 \\
P Pro & 18 & 1.96 & 1.72 \\
Q Gln & 4 & 7.73 & 7.73 \\
R Ang & 18 & 7.98 & 10.30 \\
S Ser & 24 & 6.17 & 6.87 \\
T Thr & 16 & 2.65 & 3.00 \\
V Val & 7 & 0.00 & 0.00 \\
W Trp & 0 & 1.87 & 1.29 \\
Y Tyr & 3 & 0.00 & 0.00 \\
B Asx & 0 & 0.00 & 0.00 \\
Z Glx & 0 & 0.00 & 0.00 \\
X Xxx & 0 & 0.00 & 0.43 \\
Ter & 1 & & \\
\hline & & &
\end{tabular}

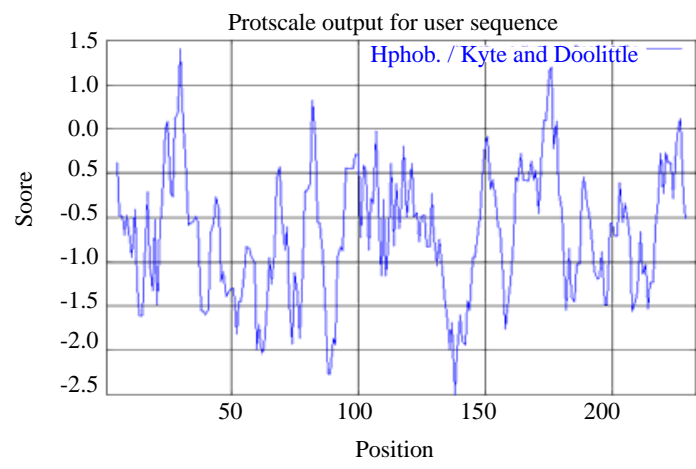

Fig. 5: Hydropathy profile of BCL-10

at residues of 1-60, 65-75, 79-116, 138-150, 163, 165-166, 175-182, 203-213 and 219-233; BCL-10 contains 14 flexible regions which can occur many distortions and can create more secondary institutions (Fig. 4).

Hydrophobicity analysis results: Hydrophobicity analysis results showed that the maximun hydrophobic index was 1.400 , the minimum hydrophobic index was -2.500 ; the average of hydrophobicity: -0.664807 which indicated that this porcine BCL-10 sequence is of asoluble protein (Fig. 5).

Transmembrane region analysis results: TMpred predicted porcine BCL-10 had twenty transmembrane regions (Only scores above 500 are considered significant (Fig. 6).

Signal peptide analysis results: Signal peptide analysis result from the SingalP showed that BCL-10 had no signal peptide (Fig. 7).

CARD structural domain analysis results: Figure 8 showed that the porcine $B C L-10$ gene had an CARD 


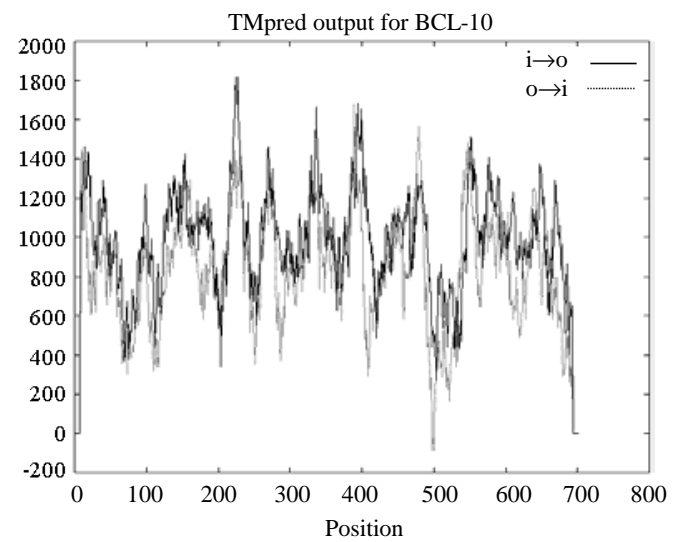

Fig. 6: Transmembrane domain prediction of porcine BCL-10

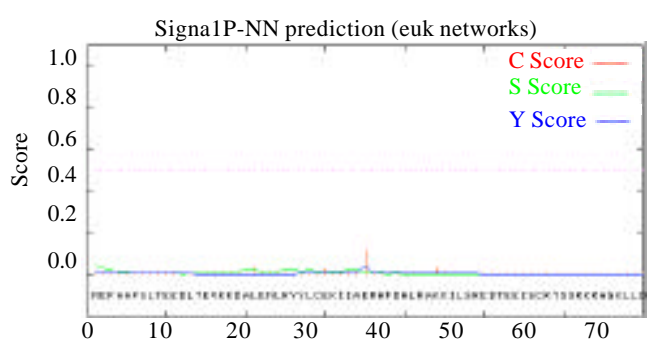

Fig. 7: Signal peptide prediction of porcine BCL-10

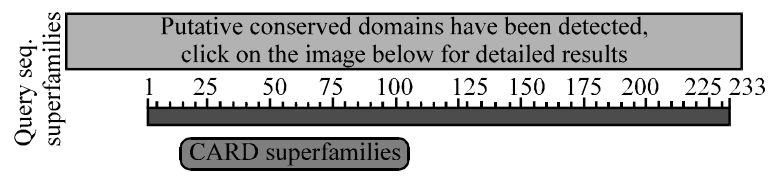

Fig. 8: CARD structural domain of BCL10

structure field which located in 15-100 amino acids. It seemed that it's the function of porcine BCL-10 was similar to the human BCL-10.

Construction of the prokaryotic expressed vector: The result of the assay of the PET32-BCL10 by double digestion showed that there was a strip in the $700 \mathrm{bp}$ (Fig. 2) which was showed that the prokaryotic expression plasmid was successfully constructed.

Optimization of the condition of prokaryotic expression and Western-blot analysis: The recombinant prokaryotic expression bacterium was induced and trained in IPTG. The results showed that there was no significant difference expression level of fusion protein in the inducing conditions of IPTG between $0.8 \sim 1.2 \mathrm{mmol} \mathrm{L}^{-1}$, so choice he proper inducing conditions of $1.0 \mathrm{mmol} \mathrm{L}^{-1}$ IPTG as the working concentration of inducing and expression. The expression level of fusion protein was maximum after induced $1 \mathrm{~h}$ in contrast, there was no

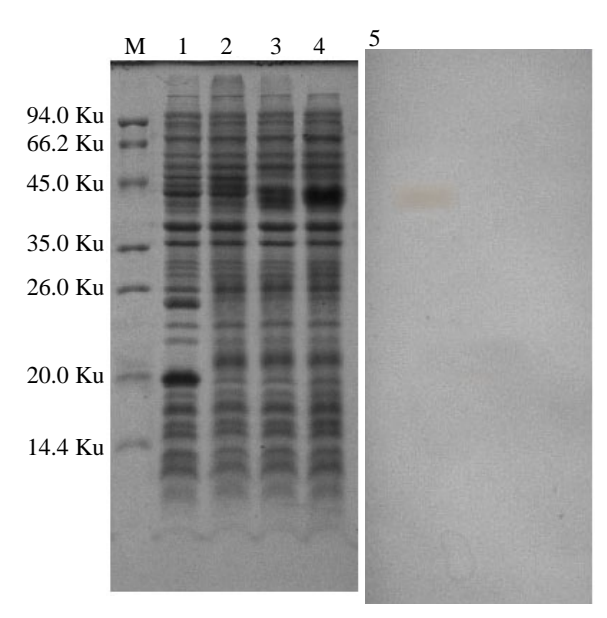

Fig. 9: SDS-PAGE and Western-Blot analysis of the recombinant protein, Lane M: Protein Marker. Lane 1: The expressed-strain (containing PET32a) induced by IPTG. Lane 2: no-induced expressed-strains. Lane 3, 4: induced expressed-strain (containing PET32-BCL10) for 1 and $5 \mathrm{~h}$. Lane 5: Western-blot analysis of the recombinant protein

significant difference expression amount in the same inducing conditions of IPT for $5 \mathrm{~h}$. The expressed fusion protein which was purified from the recombinant prokaryotic expression bacterium by ultrasonic fragmentation, in a form of inclusion body was analyzed by BandScan Software showed that interest protein accounted for $35 \%$ of total bacterial protein. There was a $45 \mathrm{Ku}$ protein appearing (Fig. 9) in cleaved product lane of induced bacterium which matched to interest protein theory value after inclusion body was washed by DOC, dissolved by SKL and preliminarily purified.

Western-blot analysis indicated that there was a special $45 \mathrm{Ku}$ protein appeared in cleaved product lane after the reaction between the induced and expressed fusion proteins of BCL-10 and mouse histidine Monoclonal antibodies (mAb) (Fig. 9).

Active detection of prokaryotic expressed fusion products: The detection showed that there was a obvious effect of cell proliferation ( $<<0.05, n=5)$ when applying fusion protein and ConA in the same dosage to lymphocyte cell line and the proliferation index was 1.418 but the effect is limited with only fusion protein or ConA.

\section{DISCUSSION}

From the research, $B C L-10$ gene is an important autarcetic functional gene of human which has a high 
expression in activating organism to produce ripe $\mathrm{B}$ lymphocyte but in different $B$ lymphocyte has a different expressed dose.

Wegener's research showed that the BCL-10 has the function of promoting $\mathrm{B}$ lymphocyte to develop by activating the molecular signal pathway protein not by directly activating (Wegener and Krappmann, 2007). From CJ Wu's study, researchers can conclude that BCL-10 activated the anti bacterial antibody to release protein of $\mathrm{NF}-\mathrm{kB}$.

This situation was also found in the process of $\mathrm{T}$ lymphocyte differentiation a nd maturation. BCL-10 gene deletion brings serious influence to the Lymphocytic action and the immunoglobulin in blood serum is in a serious shortage of level and the reaction of cellular immunity and humoral immunity will weaken obviously which will not create significant protection for the body when bacteria or virus invade body.

In this study, the homology of these base sequences of porcine BCL-10 and other species were less closer than $90 \%$ but Sequence comparison of amino acid sequence indicated that these sequences had highly homology between pig and human, $>95 \%$, their protein structure were quite similar and it seemed that the problem rests in unequal usage of codon among different species. The fused expression test showed that inclusion body had significant effects of promoting proliferation of porcine splenocyte, after it was purified, dialyzed and reannealed.

\section{CONCLUSION}

In this research, researchers successfully amplified $B C L-10$ gene from porcine peripheral-blood lymphocyte, transferred it into prokaryotic expression vector $\mathrm{PET}_{32} \mathrm{a}(+)$ and proceeded a preliminary study on prokaryotic expression which set the experimental basis for future study and research on the biologic activity and function of the BCL-10 protein and lay a foundation of further development of new porcine immunologic adjuvant.

\section{REFERENCES}

Bhattacharyya, S., A. Borthakur, S. Tyagi, R. Gill, M.L. Chen, P.K. Dudeja and J.K. Tobacman, 2009. BCL10 is required for NF- $\mathrm{KB}$ nuclear translocation by both canonical and non-canonical pathways and for NF- $k$ B-Inducing Kinase (NIK) phosphorylation. J. Biol. Chem., 15: 673-683.

Briones, J., E. Moga, I. Espinosa, C. Vergara and E. Alvarez et al., 2009. Bcl-10 protein highly correlates with the expression of phosphorylated p65 NF- $\mathrm{kB}$ in peripheral T-cell lymphomas and is associated with clinical outcome. Histopathology, 54: 478-485.

Courts, C., M. Montesinos-Rongen, J. Martin-Subero, A. Brunn and D. Siemer et al., 2007. Transcriptional profiling of the nuclear factor-kappaB pathway identifies a subgroup of primary lymphoma of the central nervous system with low BCL10 expression. J. Neuropathol. Exp. Neurol., 66: 230-237.

Guo-Quan, H., G. Wan-Zhu, W. Yin, X. Zhi-Wen and Z. Ling et al., 2009. Expression of Neijiang porcine interleukin 15 gene and study on immunologic enhancement of the expressed IL-15. Chin. Chin. Vet. Sci., 39: 543-549.

Jost, P.J. and J. Ruland, 2007. Aberrant NF-kappaB signaling in lymphoma: Mechanisms, consequences and therapeutic implications. Blood, 109: 2700-2707.

Kovar, L., J. Kopecky and B. Rihova, 2009. Salivary gland extract from ixodes ricinus tick polarizes the cytokine profile toward Th2 and suppresses proliferation of $\mathrm{T}$ lymphocytes in human PBMC culture. J. Parasitol., 87: 1342-1348.

Sagaert, X., C.D. Wolf-Peeters, H. Noels and M. Baens, 2007. The pathogenesis of MALT lymphomas: where do we stand?. Leukemia, 21: 389-396.

Wegener, E. and D. Krappmann, 2007. CARD-Bc110-Malt1 signalosomes: Missing link to NF- $\mathrm{KB}$. Sci. STKE, 384: pe21-pe21.

Zhang, Q., R. Siebert, M. Yan, B. Hinzmann and $\mathrm{X}$. Cui et al., 1999. Inactivating mutations and over expression of BCL10, a caspase recruitment domain-containing gene, in MALT lymphoma with $\mathrm{t}(1 ; 14)(\mathrm{p} 22 ; \mathrm{q} 32)$. Nat. Genet., 22: 63-68.

Zhu, L. and X. Chen, 2000. Commonly Used Experimental Methods of Immunology. People's Medical Publishing House, Bejing, China. 